Faulding have now completed trials investigating an alternate ampoule proposed for use with fentanyl citrate. This information is now under review to determine how this design may be introduced to the Canadian market. We take all comments from our customers seriously as we recognise their value in helping to improve the excellence of our products and services. As such, we appreciate the opportunity to comment on the letter and to share the actions of our company.

Scott Armour

Regulatory Affairs Associate

Faulding Canada Inc.

\section{Egress of air through minitracheostomy tube: a dilemma about its position}

To the Editor:

Retention of tracheobronchial secretions is a frequent cause of morbidity in patients with neuromuscular disorders. The advantages of minitracheostomy over more traditional methods of dealing with sputum retention have been described. ${ }^{1,2}$ We report a complication of minitracheostomy which was otherwise unsuspected.

A 29-yr-old woman was referred with progressive muscle weakness in all limbs, difficulty in swallowing and breathing and ineffective cough and was diagnosed as Guillain Barré Syndrome based on nerve conduction studies. After tracheal intubation and pressure support ventilation, she showed rapid motor recovery but was unable to clear her respiratory secretions. Extubation and minitracheostomy were planned.

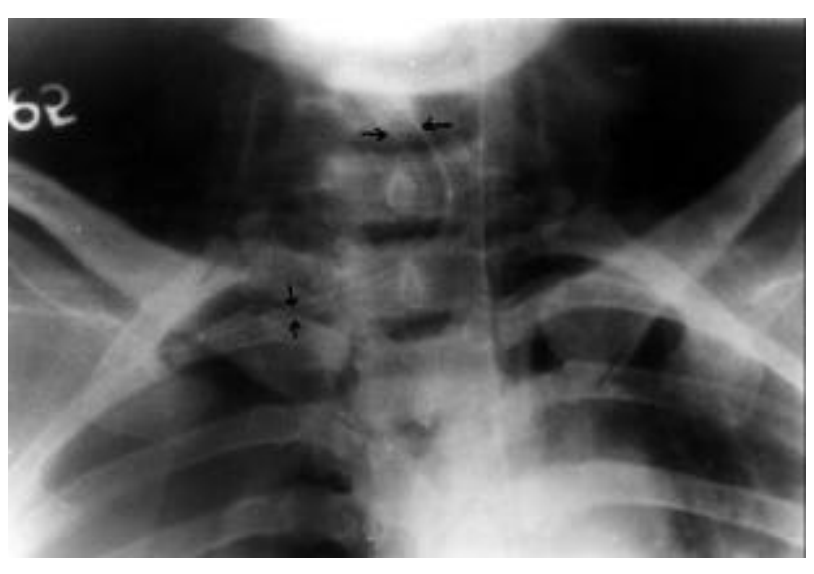

FIGURE -Position of minitracheostomy and right side pneumothorax in chest Ray AP view.
Al6-gauge needle was introduced between lst and 2nd tracheal rings, air aspirated and a guide wire passed, the latter requiring some force. The internal diameter tracheal cannula was then slid over the guide wire and fixed in position. The position was checked by aspirating air through the minitracheostomy tube and, with every breath, air moved freely in and out of the tube. However no secretions could be aspirated even after instilling saline. Chest $\mathrm{X}$ ray revealed the cannula tip in the right pleural space and right side pneumothorax with collapsed lung parenchyma (Figure). The cannula was removed and the patient was managed with a nasotracheal tube.

Chest X-ray usually confirms the proper position of tracheostomy. In cases where negotiation of the guide wire is not smooth, the absence of tracheal secretion should raise suspicion about the malposition of tracheostomy tube.

Chandra Kant Pandey MD

Devashish Chakravarty MD PDCC

Namita Singh MD PDCC

Rehana Sikora MD

Lucknow, India

\section{References}

1 Matthews HR, Hopkinson RB. Treatment of sputum retention by minitracheotomy. Br J Surg 1984; 71: $147-50$.

2 Yeoh NTL, Wells FC, Goldstraw P. A complication of minitracheostomy. Br J Surg 1985; 72: 633. 